\title{
Reprint
}

\section{Analytical Computation of Multipath Components in the indoor power grid}

\author{
D. Anastasiadou and T. Antonakopoulos
}

IEEE International Symposium on Circuits and Systems, ISCAS-2003

\author{
BANGKOK, THAILAND, MAY 2003
}

\begin{abstract}
Copyright Notice: This material is presented to ensure timely dissemination of scholarly and technical work. Copyright and all rights therein are retained by authors or by other copyright holders. All persons copying this information are expected to adhere to the terms and constraints invoked by each author's copyright. In most cases, these works may not be reposted or mass reproduced without the explicit permission of the copyright holder.
\end{abstract}




\title{
ANALYTICAL COMPUTATION OF MULTIPATH COMPONENTS IN THE INDOOR POWER GRID
}

\author{
Despina Anastasiadou and Theodore Antonakopoulos \\ University of Patras, Department of Electrical Engineering and Computers Technology, \\ 26500 Rio, Patras, Greece \\ Computers Technology Institute - CTI, Riga Feraiou 61, 26100 Patras, Greece
}

\begin{abstract}
Varying impedance loads connected to the termination points of power-line channels result in variable channel behavior. Any transmitted signal in such a communications system is subject to time-varying multipath fading. In this paper we present a method for determining the multipath components of any transmitterreceiver pair in the indoor power-line environment. Such information could be valuable in the process of designing the proper receiver architecture. The proposed method is applied to a network to demonstrate its usefulness in explaining the network's time-dependent behavior.
\end{abstract}

\section{INTRODUCTION}

The increasing demand for broadband telecommunication services in today's Small Office/Home Office (SOHO) environment has stirred up interest in alternative communications media. The indoor power grid can be considered a prominent candidate, with its main advantages resulting from the utilization of the existing infrastructure and the abundance of its access points, in the form of AC outlets. However, prior to the development of viable power-line systems for telecommunication services, analytical and/or experimental-based statistical modeling of this time-varying channel is required.

The power-line channel differs considerably in topology, structure and physical properties from conventional media such as twisted pair, coaxial and fiber-optical cables, exhibiting rather hostile characteristics. Initially designed for power distribution, the power grid is far from optimized for high-speed data transmission. Common causes of impairment include time and frequency varying impedances and considerable noise due to loads connected to its outlets. As a result, variable levels of signal attenuation are observed on the channel [1].

Various measurement-based and analytical models have been proposed in literature, concerning the communication properties of the indoor power grid. The most common approach considers the channel as a 'black box' and extracts a number of system parameters using extensive experimental results [2], [3]. Statistical modeling of the measured additive noise and the channel transfer function has also been attempted, without producing a widely accepted channel model. Such results fail to explain the cause of the time-dependent behavior of the physical channel.

Modeling the power-line for high-speed communications can be aided by regarding the medium as a multipath channel [3]. The multipath nature of the power grid arises from the presence of several braches and impedance mismatches, causing numerous reflections of the transmitted signal. Analytical calculation of the multipath effect in the indoor power grid is feasible due to its loop-free topology and its limited complexity. Thus, the multipath effect can be traced back to the channel physical characteristics by calculating cable loss, reflection and transmission factors. The method presented in this paper is used to determine the significant transmission paths that contribute to the time and frequency variable behavior between any given transmitter-receiver pair of an arbitrary indoor power-line network.

This paper is organized as follows: Section 2 defines the channel response, its multipath components and the minimum information required for describing the physical characteristics of an indoor power-line network. Section 3 presents the algorithm for calculating the reflection coefficients at any network point and the complex reflection factor of every path. Finally, Section 4 gives an example, demonstrating how the proposed method can be used to determine the multipath components between any pair of communicating devices.

\section{MULTIPATH EFFECTS AND REFLECTION COEFFICIENTS}

Multipath fading is the result of signal interference among several delayed replicas of a transmitted signal, having variable amplitude and phase. High frequency signal propagation in power-line networks is performed though multiple paths, one of which is the 'direct' (shortest) path between the transmitter and the receiver. Therefore, the received signal is composed of numerous multipath components, which are distorted copies of the transmitted signal. Amplitude and phase distortion is caused by line propagation and channel discontinuities. Such discontinuities appear due to load mismatching at termination points (where loads are connected) and network nodes (where branches begin).

Transmission of an ideal wideband signal, such as the impulse function $\delta(t)$, through a multipath environment with $L$ paths results in a train of delayed impulses with different strengths at the receiver [4]. Modifying the general expression, to suit the case of an indoor power-line communication network, the channel response is given by:

$$
h(\tau, t)=\sum_{i=1}^{L}\{\underbrace{r_{i}}_{\begin{array}{c}
\text { reflection } \\
\text { factor }
\end{array}} \cdot \underbrace{e^{j \gamma l_{i}}}_{\begin{array}{c}
\text { prapagation } \\
\text { factor }
\end{array}} \cdot e^{j \phi_{i}} \cdot \delta\left(t-\tau_{i}\right)\}
$$


where $r_{i}$ is the complex reflection factor of path $i, \gamma$ is the propagation factor, and $\phi_{i}=2 \pi f \tau_{i}$, where the delay $\tau_{i}=l_{i} / v$ depends on the path's length $l_{i}$ and group velocity of propagation $v$. The reflection factor $r_{i}$ is the product of all reflectiontransmission coefficients $\rho_{i(1 . . k)}$ of path $i$ when propagating through $k$ discontinuities, and is given by:

$$
r_{i}=\prod_{j=1}^{k} \rho_{i j}
$$

Therefore, in order to determine the channel impulse response, or the equivalent frequency response, the significant transmission paths have to be determined along with all the reflection coefficients.

\subsection{Network description}

The indoor power grid can be described using the following parameters: termination impedances, line sections types and lengths. For each type of cable, the characteristic impedance $Z_{0}$ and the propagation factor $\gamma$ of the line can be calculated through transmission line theory equations, using the cable primary parameters $\left(R^{\prime}, L^{\prime}, C^{\prime} G^{\prime}\right)[5]$.

Network description matrices comprise information such as the number of termination points and nodes, their connectivity, line section lengths and cable characteristics $\left(Z_{0}\right.$ and $\left.\gamma\right)$. Consider a network with $m$ termination points $\mathrm{T}_{\mathrm{i}}$ and $n$ nodes $\mathrm{C}_{\mathrm{j}}$, which is described by:

1. The matrix $\boldsymbol{T C}\left[m_{x} n\right]$, which describes the interconnections between termination points and nodes. Each line corresponds to a termination point $\mathrm{T}_{\mathrm{i}}$ and each column to a node $\mathrm{C}_{\mathrm{j}}$. Element $\mathrm{TC}(\mathrm{i}, \mathrm{j})$ is equal to unity when a connection between $\mathrm{T}_{\mathrm{i}}$ and $\mathrm{C}_{\mathrm{j}}$ exists, or zero otherwise.

2. The matrix $\boldsymbol{C} \boldsymbol{C}[n \times n]$, which describes the interconnections between nodes. Each line and each column correspond to a certain node $\mathrm{C}_{\mathrm{i}}$. Unit elements $\mathrm{CC}(\mathrm{i}, \mathrm{j})=1$ imply a connection between the corresponding nodes, whereas $\mathrm{CC}(\mathrm{i}, \mathrm{j})=0$ implies no direct connection. Since nodes cannot be connected to themselves and $\mathrm{C}_{\mathrm{i}} \mathrm{C}_{\mathrm{j}}=\mathrm{C}_{\mathrm{j}} \mathrm{C}_{\mathrm{i}}$, the matrix has a zero diagonal, and exhibits symmetry around it.

3. The matrix $\boldsymbol{L}_{\boldsymbol{T C}}\left[m_{x} n\right]$, which is generated by the $\boldsymbol{T C}$ matrix when its unit elements are replaced by the corresponding lengths $l_{T i C j}$.

4. The matrix $\boldsymbol{L}_{\boldsymbol{C} C}\left[n_{x} n\right]$, which is generated by the $\boldsymbol{C} \boldsymbol{C}$ matrix when its unit elements are replaced by the corresponding lengths $l_{C i C j}$. The $\boldsymbol{L}_{\boldsymbol{C} C}$ matrix is also symmetric around its diagonal.

5. The matrix $\boldsymbol{Z}_{T}\left[\begin{array}{lll}m & x & 1\end{array}\right]$, where each line element is the termination impedance of the corresponding termination point:

$$
Z_{T}=\left[\begin{array}{llll}
Z_{1} & Z_{2} & \ldots & Z_{m}
\end{array}\right]^{\mathrm{T}}
$$

The above network description, along with $\mathrm{Z}_{0}$ and $\gamma$, can be used as input to the algorithm that calculates the reflection coefficients at each point in the network according to the following steps:

- $\quad$ Determine the ABCD matrix for each line section, using $Z_{0}, \gamma$ and section's length $l$,
- Calculate the equivalent impedances at each node, depending on the direction of the signal (Figure 1), and

- Calculate the reflection coefficients at each network point, according to:

$$
\rho_{x y}=\frac{Z_{x y}-Z_{0}}{Z_{x y}+Z_{0}}
$$

where $\mathrm{x}, \mathrm{y}$ indices denote signal propagation from point $\mathrm{y}$ to $\mathrm{x}$. The reflected signal travels back to point $y$.

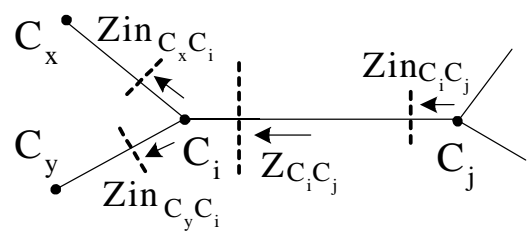

Figure 1. Node impedances for signal propagation from node $\mathrm{C}_{\mathrm{j}}$ to node $\mathrm{C}_{\mathrm{i}}$.

\section{The Reflection Coefficients Algorithm}

The algorithm described in this section produces the reflection coefficients at each point of discontinuity on the channel, namely termination points and nodes of the network. Since the algorithm is intended for the analysis of the indoor power grid, certain general conditions are always valid:

- $\quad$ Each termination point $T_{i}$ is connected to a single node.

- Connections between termination points do not exist,

- $\quad$ Nodes do not form loops, since the network has a radial topology.

\subsection{Impedance calculation}

In order to calculate the reflection coefficients at any point in the network, the corresponding impedances have to be derived. Throughout this text the following name conventions are used:

- $\quad$ Termination impedance $Z_{T i}$, is the impedance of the load connected at the termination point $\mathrm{T}_{\mathrm{i}}$,

- Equivalent impedance $Z_{C i X j}$, is the combined impedance at node $C_{i}$ for a signal traveling from $X_{j}$ towards $C_{i}$.

- Input impedance $\operatorname{Zin}_{X i X j}$, is the transformation of $\mathrm{Z}_{\mathrm{XiXj}}$ along the line from point $\mathrm{X}_{\mathrm{j}}$ to $\mathrm{X}_{\mathrm{i}}$, according to:

$$
\operatorname{Zin}_{X X}(i, j)=\frac{A_{X_{i} X_{j}} \cdot Z_{X X}(i, j)+B_{X_{i} X_{j}}}{C_{X_{i} X_{j}} \cdot Z_{X X}(i, j)+D_{X_{i} X_{j}}}
$$

where $\boldsymbol{A} \boldsymbol{B} \boldsymbol{C} \boldsymbol{D}_{X i X j}$ is the $\mathrm{ABCD}$ matrix of the line section $\mathrm{X}_{\mathrm{i}} \mathrm{X}_{\mathrm{j}}[6]$. Before proceeding with impedance calculations, the $\boldsymbol{A} \boldsymbol{B} \boldsymbol{C} \boldsymbol{D}_{T C}$ and $\boldsymbol{A B C} \boldsymbol{D}_{\boldsymbol{C} C}$ matrices are calculated. The matrix $\boldsymbol{A} \boldsymbol{B C} \boldsymbol{D}_{\boldsymbol{T C}}$ is calculated using matrix $\boldsymbol{T C}$, by substituting each unit element with the ABCD sub-matrix of the corresponding line section, as shown in (6). The same procedure is used for calculating matrix $\boldsymbol{A B C} \boldsymbol{D}_{\boldsymbol{C C}}$ using matrix $\boldsymbol{C C}$.

$$
A B C D_{T C}(i, j)=\left[\begin{array}{ll}
A & B \\
C & D
\end{array}\right]_{i j}=\left[\begin{array}{ll}
A_{T i C j} & B_{T i C j} \\
C_{T i C j} & D_{T i C j}
\end{array}\right]
$$

In order to calculate the network impedances, the following process is used: 
Step 1: Calculate the input impedance matrix $\operatorname{Zin}_{T C}\left[m_{x} n\right]$, formed by elements $\operatorname{Zin}_{T C}(i, j)$ that represent the input impedance at node $C_{j}$ for a signal traveling from $C_{j}$ to termination point $T_{i}$. The matrix is generated by replacing the unit elements of $\boldsymbol{T C}$ matrix with the corresponding impedances determined by (5) when $\mathrm{X}_{\mathrm{i}} \mathrm{X}_{\mathrm{j}}=\mathrm{T}_{\mathrm{i}} \mathrm{C}_{\mathrm{j}}$ and $\mathrm{Z}_{\mathrm{XX}}(\mathrm{i}, \mathrm{j})=\mathrm{Z}_{\mathrm{T}}(\mathrm{i})$.

Step 2: Calculate the input and the equivalent impedance matrices at any node. The input impedance matrix $\operatorname{Zin}_{C C}[n \times n]$ is formed by elements $\operatorname{Zin}_{C C}(i, j)$ which represent the input impedance at node $C_{j}$ for signal propagation from $C_{j}$ to $C_{i}$, calculated using (5), with $X_{i} X_{j}=C_{i} C_{j}$ and $Z_{X X}(i, j)=Z_{C C}(i, j)$. Equivalent impedance matrix $\boldsymbol{Z}_{C C}[n \times n]$ is formed by elements $Z_{C C}(i, j)$ which represent the equivalent impedance at connection point $C_{i}$ as seen from $C_{j}$. $Z_{C C}(i, j)$ is the parallel combination of all input impedances at $C_{i}$ as seen by a signal traveling from $C_{i}$ to every adjacent point, except $C_{j}$ :

$$
Z_{C C}(i, j)=/ /(\underbrace{\operatorname{Zin}_{T C}(r, i)}_{\forall r \in[1, m]}, \underbrace{\operatorname{Zin}_{C C}(s, i)}_{\forall s \neq j \in[1, n]})
$$

Both matrices are generated by replacing the unit elements of matrix $\boldsymbol{C} \boldsymbol{C}$ with the corresponding impedances. Equations (5) and (7) indicate that the calculation of $\boldsymbol{Z}_{\boldsymbol{C C}}$ requires $\boldsymbol{Z i n}_{\boldsymbol{C C}}$ and vice versa, therefore, simultaneous calculation of both matrices has to be performed. Since all required elements are not known prior to calculation, a computationally efficient process was developed in order to determine the next element that can be calculated using those already available. A detailed description of this process is given in the next subsection.

Step 3: The last step involves calculation of the equivalent impedance matrix $\boldsymbol{Z}_{C T}\left[m_{x} n\right]$, formed by $Z_{C T}(i, j)$ elements, which represent the equivalent impedance at node $C_{i}$. This impedance is the parallel combination of all input impedances at $C_{i}$ as seen by a signal traveling from $C_{i}$ to every adjacent point, except $T_{j}$ :

$$
Z_{C T}(i, j)=/ /(\underbrace{\operatorname{Zin}_{C C}(r, i)}_{\forall r \in[1, n]}, \underbrace{\operatorname{Zin}_{T C}(s, i)}_{\forall s \neq j \in[1, m]})
$$

This matrix is identical to the transposed $\boldsymbol{T C}$ matrix $\left(\boldsymbol{T C} \boldsymbol{C}^{\boldsymbol{T}}\right.$, generated by substituting its unit elements with the corresponding impedance $\left(\mathrm{CT}^{\mathrm{T}}(\mathrm{i}, \mathrm{j}) \rightarrow \mathrm{Z}_{\mathrm{CT}}(\mathrm{i}, \mathrm{j})\right)$.

\subsubsection{Concurrent computation of the two impedance matrices}

As already mentioned, the $\boldsymbol{Z i n}_{\boldsymbol{C C}}$ and $\boldsymbol{Z}_{\boldsymbol{C C}}$ impedance matrices have to be computed simultaneously, since all required elements are not known prior to calculation. The proposed process estimates the next element that can be calculated from those already available and has to be used in order to avoid any unnecessary or unsolvable impedance calculation steps. The process is based on the rearrangement of the $\boldsymbol{T C}, \boldsymbol{C C}$ and all corresponding matrices according to the following rule:

The smallest indices of the new $\boldsymbol{C} \boldsymbol{C}$ matrix correspond to 'outer nodes', whereas the largest indices to 'inner nodes'. The criterion of characterizing a node as 'inner' or 'outer' is based on the sum of its distances from all other nodes. The term distance refers to the number of intermediate nodes that can be found in the shortest path between the two nodes. The node with the smallest sum of distances is considered to be the central node. This new order of node indices is used to rearrange all other matrices. The $\boldsymbol{C C}$ matrix remains symmetric around its zero diagonal. Keeping the name convention, element $(\mathrm{i}, \mathrm{j})$ denotes signal propagation from $X_{j}$ to $X_{i}$. The proposed process calculates the $\boldsymbol{Z}_{\boldsymbol{C C}}$ and $\boldsymbol{Z i n}_{\boldsymbol{C C}}$ elements based on the signal direction implied by their indices:

- Elements above the diagonal $(i>j)$ indicate direction from inner to outer nodes,

- Elements beneath the diagonal $(i<j)$, indicate direction from outer to inner nodes.

For each node $C_{i}$, impedances that correspond to one direction depend only on elements of the same direction (Figure 1). Thus, the calculation of elements above the diagonal of the new $\boldsymbol{C C}$ matrix requires knowledge only of the elements above the diagonal, and calculation of elements beneath the diagonal requires only elements beneath it. Furthermore, it can be proven that there is only one non-zero element per column beneath the diagonal. This statement is true, since for each node $C_{j}$ connected to $s_{j}$ other nodes, there is 1 element corresponding to one direction and $s_{j}-1$ elements to the other. By symmetry, only one non-zero element per line is located above the diagonal.

Following the above procedure, $\boldsymbol{Z}_{\boldsymbol{C C}}$ and $\boldsymbol{Z i n}_{\boldsymbol{C}}$ matrices can be computed using (7) in the more efficient manner. The process starts with the calculation of each line of the matrices above the diagonal, beginning with line $i=1$, and progresses to the calculation of each column of the matrices beneath the diagonal, beginning with column $j=n-1$.

\subsection{Reflection coefficient calculation}

Using the computed impedance matrices, the reflection coefficients at any point in the network can be calculated. Moreover, each reflection coefficient at node $C_{i} \quad\left(\rho_{C i X j}\right)$ corresponds to a number of transmission coefficients, one for each line section, between $C_{i}$ and every adjacent node $\mathrm{X}_{\mathrm{i}} \neq \mathrm{X}_{\mathrm{j}}$. They are all equal to each other and are given by:

$$
\tau_{C i \mathrm{X} j}=1+\rho_{C i \mathrm{X} j}
$$

Then, the following reflection matrices are calculated:

1. reflection coefficient matrix $\boldsymbol{\rho}_{T C}[m \times n]$, which is created by replacing all unit elements of $\boldsymbol{T C}$ with the corresponding coefficients $\rho_{T C}(i, j)$, calculated using (4) with $Z_{x y}=Z_{T}(i)$.

2. reflection coefficient matrix $\rho_{C C}[n \times n]$, which is created by replacing all unit elements of $\boldsymbol{C C}$ with the corresponding coefficients $\rho_{C C}(i, j)$, calculated using (4) with $Z_{x y}=Z_{C C}(i, j)$.

3. reflection coefficient matrix, $\rho_{C T}[n \times n]$, which is created by replacing all unit elements of $\boldsymbol{T} \boldsymbol{C}^{\boldsymbol{T}}$ with the corresponding coefficients $\rho_{C T}(i, j)$, calculated using (4) with $Z_{x y}=Z_{C T}(i, j)$.

Using the reflection coefficient matrices, the complex reflection factor and the channel response of any path is computed.

\section{Sample network and simulation results}

The effectiveness of the method is demonstrated by applying it to a representative example, shown in Figure 2. The wiring cable is a typical residential cable (H05VVF, $3 \times 1.5 \mathrm{~mm}^{2}$ ) and its characteristics $\left(Z_{0}, \gamma\right)$ have been calculated in [7]. 


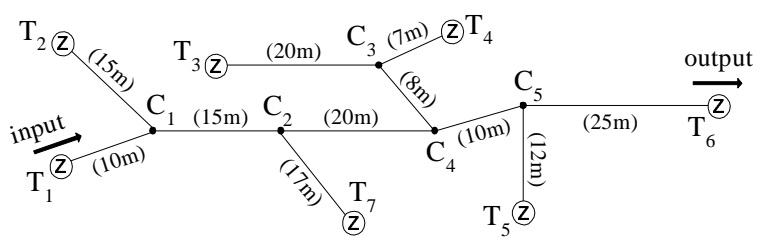

Figure 2. Example of an indoor power-line network.

The connectivity matrices $\boldsymbol{T C}[8 x 6]$ and $\boldsymbol{C} \boldsymbol{C}[6 x 6]$ are given by:

$$
T C=\left[\begin{array}{lllll}
1 & 0 & 0 & 0 & 0 \\
1 & 0 & 0 & 0 & 0 \\
0 & 0 & 1 & 0 & 0 \\
0 & 0 & 1 & 0 & 0 \\
0 & 0 & 0 & 0 & 1 \\
0 & 0 & 0 & 0 & 1 \\
0 & 1 & 0 & 0 & 0
\end{array}\right] \quad C C=\left[\begin{array}{lllll}
0 & 1 & 0 & 0 & 0 \\
1 & 0 & 0 & 1 & 0 \\
0 & 0 & 0 & 1 & 0 \\
0 & 1 & 1 & 0 & 1 \\
0 & 0 & 0 & 1 & 0
\end{array}\right]
$$

Consider a transmitter at point $T_{1}$ and a receiver at point $T_{6}$. Signal propagation between these points is done through a large number of paths. Each path corresponds to a path reflection coefficient given by (2), derived here for the shortest path $\left(\mathrm{T}_{1} \mathrm{C}_{1} \mathrm{C}_{2} \mathrm{C}_{4} \mathrm{C}_{5} \mathrm{~T}_{6}\right)$ :

$$
\begin{aligned}
& \rho_{1}=\tau_{C_{1} T_{1}} \tau_{C_{2} C_{1}} \tau_{C_{4} C_{2}} \tau_{C_{5} C_{4}} \tau_{T_{6} C_{5}} \\
& l_{1}=l_{T_{1} C_{1}}+l_{C_{1} C_{2}}+l_{C_{2} C_{4}}+l_{C_{4} C_{5}}+l_{C_{5} T_{6}}
\end{aligned}
$$

where $\rho_{l}$ is the reflection factor and $l_{l}$ is the length of path 1.The corresponding propagation factor is $e^{\gamma l_{1}}$. Using (1), the channel response of the path is computed as the sum of all multipath components. By applying the previously described method for different termination impedances, we calculated the magnitude and phase of the multipath components received at point $\mathrm{T}_{6}$ and the channel frequency response, in the frequency range of 1$10 \mathrm{MHz}$. The power of the first three signal components arriving at $\mathrm{T}_{6}$ are displayed in Figure 3.
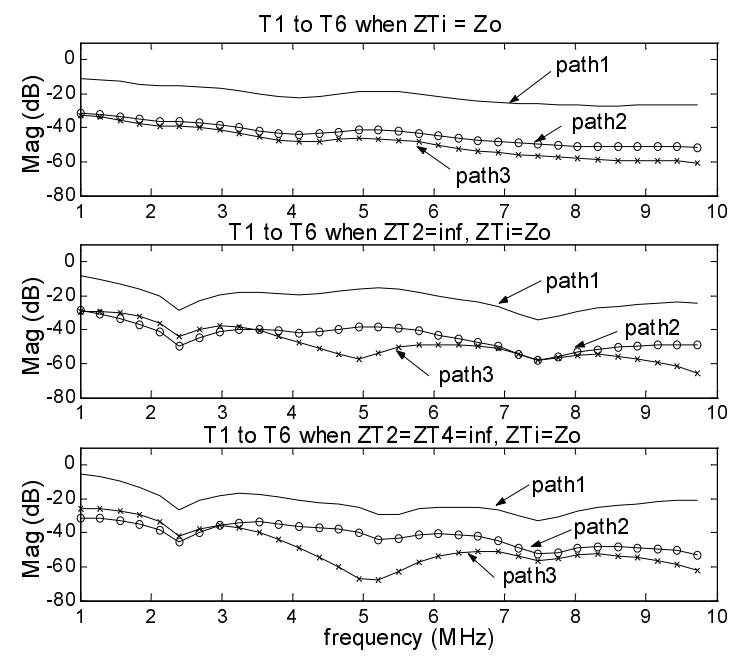

Figure 3. Power magnitude of the first three multipath components for different termination loads.

The three curves correspond to: a) matched termination loads at all points $\left.\left(Z_{o}\right), b\right)$ matched loads at all points except $T_{2}$, which is open circuited and c) matched loads at all points except $T_{2}$ and
$\mathrm{T}_{4}$, which are open circuited. The magnitude of the power spectrum (power of the frequency response) for these three cases is displayed in Figure 4.

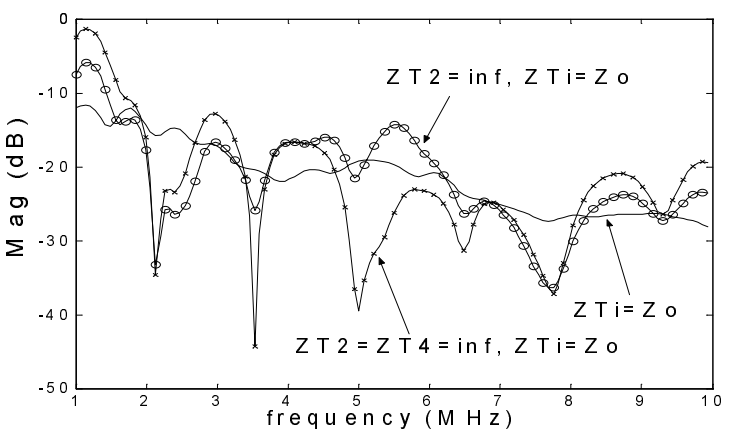

Figure 4. Magnitude of the power spectrum for different termination loads.

\section{SUMMARY}

In this work we presented a computationally efficient method for determining the multipath transmission components in indoor power-line networks. In the residential power-line environment, a limited number of termination loads (i.e. household appliances) can be found and their input impedances can be measured and characterized. Using this information and the method described in this paper the response of any power-line topology can be determined. The application of different load impedances can explain the varying channel behavior.

\section{REFERENCES}

[1] Liu D., Flint B., Gaucher B., Kwark Y., "Wide Band AC Power Line Characterization", IEEE Trans. on Consumer Electronics, Vol. 45, No. 4, Nov 1999, pp. 1087-1097.

[2] Philips H., "Modeling of Powerline Communication Channels", Proceedings of the $3^{\text {rd }}$ International Symposium on Power-Line Communications and its Applications, Lancaster, UK, 1999, pp. 14-21.

[3] Zimmermann M. and Dostert K., "A MultiPath Model for the PowerLine Channel", IEEE Transactions on Communications, Vol. 50, No. 4, April 2002, pp. 553-559.

[4] Pahlavan K. and Levesque A., Wireless information Networks, Wiley Interscience, New York, 1995

[5] Matick R.E., Transmission Lines for Digital and Communication Networks, IEEE Press, New York, 1995.

[6] Chen W.K., The Circuits and Filters Handbook, IEEE-CRC Press, Chicago, 1995.

[7] Anastasiadou D. and Antonakopoulos T., “An Experimental Setup for Characterizing the Residential Power Grid Variable Behavior", Proceedings of the $6^{\text {th }}$ International Symposium on Power-Line Communications and its Applications, Athens, Greece, 2002, pp. 65-70.

This work was partially supported by the 'Karatheodoris' R\&D program of the University of Patras and project 00BE23 entitled 'High Speed Transmission Technology over Residential Power-Lines' of the Greek Ministry of Industry. 